\title{
Experience of Surgical Management of Gastrointestinal Stromal Tumors at a Tertiary Hospital of Nepal
}

\author{
Dhruba Narayan Sah ${ }^{*}$, Ramesh Singh Bhandari, Paleswan Joshi Lakhey, Yogendra Prasad Singh, \\ Pradeep Vaidya, Prasan Bir Singh Kansakar, Bikal Ghimire, Bishnu Prasad Kandel, \\ Jayant Kumar Sah
}

Surgical Gastroenterology, Tribhuvan University Teaching Hospital, Kathmandu, Nepal

\section{Email address:}

drshah_dn@live.com (D. N. Sah), rsbhandari09@gmail.com (R. S. Bhandari), lakheypj@gmail.com (P. J. Lakhey), yp_singh@hotmail.com (Y. P. Singh), praidya@yahoo.com (P. Vaidya), pbskansakar@hotmail.com (P. B. S. Kansakar), bikalghimire@gmail.com (B. Ghimire),drkandel@hotmail.com (B. P. Kandel), jayantsah@hotmail.com (J. K. Sah)

${ }^{*}$ Corresponding author

\section{To cite this article:}

Dhruba Narayan Sah, Ramesh Singh Bhandari, Paleswan Joshi Lakhey, Yogendra Prasad Singh, Pradeep Vaidya, Prasan Bir Singh Kansakar, Bikal Ghimire, Bishnu Prasad Kandel, Jayant Kumar Sah. Experience of Surgical Management of Gastrointestinal Stromal Tumors at a Tertiary Hospital of Nepal. Journal of Surgery. Vol. 7, No. 4, 2019, pp. 104-109. doi: 10.11648/j.js.20190704.14

Received: June 2, 2019; Accepted: July 1, 2019; Published: July 12, 2019

\begin{abstract}
Gastrointestinal stromal tumors (GISTs) are rare malignancies but a most common mesenchymal tumor. A multidisciplinary team approach is the optimal care of GIST patients after the remarkable outcomes with the development of molecular-targeted therapy. The objective is to determine the clinic-pathological spectrum and risk category of GIST along with perioperative outcomes. This is a retrospective review of GIST patients between September 2015 - August 2018 at Tribhuvan University Teaching Hospital (TUTH), Kathmandu, Nepal. Patients' clinical data, histopathology, immunohistochemistry (IHC) and outcomes were recorded and analyzed. A total of 42 GIST patients were identified with age (19-81 years) and $69 \%$ were males. Stomach $(35.7 \%)$ was the most common site followed by small bowel (23.8\%) and duodenum (14.3\%). Pain (40.5\%) followed by bleeding (30.9\%) were major indications. Max tumor dimensions were ranging from $2.8-30 \mathrm{~cm}$ and median mitotic figures were $3(0-35)$. Patients were stratified as high, intermediate, low and very low risk $(36 \%, 32 \%, 32 \%$, and $0 \%$ respectively). The majority were managed surgically out of which three cases underwent preoperative angioembolization. CD 117 was positive in $90.5 \%$. Neoadjuvant imatinib given in 2 cases while adjuvant imatinib given in 18 cases. Over a median follow up of 18 months four patients expired while 32 had no issues. Surgical resection is the preferred choice of treatment with or without the molecular targeted drug therapy. Most tumors fall in the high-risk category.
\end{abstract}

Keywords: Gastrointestinal Stromal Tumor (GIST), TUTH, Imatinib

\section{Introduction}

Gastrointestinal stromal tumor (GIST) is the most common mesenchymal tumor $(80 \%)$ of the gastrointestinal (GI) tract [1]. Although any organ in the GI tract can be involved, the stomach and small bowel are most commonly involved [2]. GISTs rarely occur in extra-gastrointestinal sites such as the omentum, mesentery, pelvis, and retroperitoneum [3]. Most of the cases are usually asymptomatic but if present symptomatically they can present with bleeding, anemia, abdominal distension, abdominal pain, or peritonitis [4].

Prediction of malignant potential of GIST is very difficult because of its wide variability in biological behaviors [5]. GISTs occurring outside of the stomach are associated with a higher malignancy potential. Because of high malignant potential, curative surgical resection should be attempted whenever tumor is suggestive of GIST [6,7]. Following curative surgery for localized GIST, patients should be categorized in risk groups for the development of recurrence. Location of tumor, size, and mitotic rate are the most important prognostic parameters [8]. These factors form the basis for consensus risk classification.

Surgery is the treatment of choice for patients with localized or potentially resectable GISTs [7]. Ro excision is 
the goal with functional preservation whenever possible. Transcatheter arterial embolization (TAE) is a safe and effective method for controlling GI bleeding from the GIST [9]. A multidisciplinary team approach is the optimal care of GIST patients after the remarkable outcomes with the development of molecular-targeted therapy (imatinib). Early diagnosis (early GISTs without metastasis) with early surgical resection is the only promising way to obtain a complete cure for this disease [10].

With the widespread use of imaging modalities for other reason, even smaller asymptomatic tumors are being picked up more frequently. Also achieving the goal of R0 surgery along with the possibility of organ-preserving, function preserving with acceptable perioperative outcomes and long term recurrence-free survival are some of the challenges which need to be addressed. Besides imatinib as targeted therapy, development of other newer drugs is promising in managing GIST. There is a sparse number of the published article from our countries regarding GIST, so it becomes necessary to analyze our current management strategies and compared with published articles worldwide. Managing a case as per evidence-based practice guidelines and able to achieve optimal outcomes is a challenge in resource-limited countries.
Aim of the study is to describe the clinicopathologic features, risk categories, and perioperative short term \& long term outcomes at TUTH.

\section{Materials and Method}

This is a retrospective review of a prospectively collected database of a total of 42 GIST patients diagnosed pathologically between Aug 2015 - Aug 2018 over 36 months at the Department of GI \& General Surgery, TU Teaching Hospital, Kathmandu, Nepal. Diagnosis of "GIST" was confirmed by histopathological examination which was further supplemented by immunohistochemistry (IHC) assay.

Patients' demographics, clinical data, investigations, operative details, histopathology (included tumor location, gross appearance, tumor size (maximum diameter in $\mathrm{cm}$ ), mitotic figures), immunohistochemistry (IHC), prognostic groups and outcomes (the perioperative and long term on follow up as much as possible were noted. Patients were followed up in outpatient in regards to readmission, adjuvant therapy and development of recurrences. The tumors were categorized into very low, low, intermediate and high-risk groups according to the modified NIH risk classification criteria (Table 1) [5, 7].

Table 1. Risk classification of GISTs (modified NIH).

\begin{tabular}{llll}
\hline Risk classification & Tumor size (cm) & Mitotic rate per 50 hpf & Tumor site \\
\hline Very Low risk & $<2$ & $\leq 5$ & Any \\
Low risk & $2.1-5.0$ & $\leq 5$ & Any \\
& $2.1-5.0$ & $>5$ & Gastric \\
Intermediate risk & $<5$ & $6-10$ & Any \\
& $5.1-10$ & $\leq 5$ & Gastric \\
& Any & Tumor rupture & Any \\
High risk & $>10$ & Any & Any \\
& Any & $>10$ & Any \\
& $2.1-5.0$ & $>5$ & Non gastric \\
& $5.1-10.0$ & $\leq 5$ & Non gastric \\
\hline
\end{tabular}

$h p f=$ high power field.

Data were reported as percentages, mean \pm standard deviation, and median and range, when applicable. Data analysis was carried out using the statistical package for social sciences version 23 (SPSS) and Microsoft Excel 2016.

\section{Results}

Table 2. Details of patients of Gastrointestinal stromal tumor (42 cases).

\begin{tabular}{ll}
\hline Clinical Characteristics & \\
Age & Median - 52 years (Range: 19-81) \\
Sex & $69 \%$ Male \\
Pain (17), Bleeding (13), Obstruction (4), Lump (4), & Incidental (3) \& others (1) \\
Indication of intervention & Median - 45 days (range 2-730) \\
Duration of symptoms & CECT (13), Exploratory Laparotomy (12), Endoscopy (12), \\
Diagnostic modality & Trucut Biopsy (5), Postoperative HPE (1) \\
Tumor Characteristics & Gastric (35.7\%), Small Bowel (23.8\%), Duodenum (14.3\%), \\
Site & Large Bowel (7.1\%), Omentum (9.5\%), Others $(9.6 \%)$ \\
& $19.1 \%$ e-gist (Extra gastrointestinal) \\
Max Tumor Dimension (28) & Mean \pm S. D - 7.2 \pm 5.2cm (range 2.8 -30$)$ \\
& $2-5 \mathrm{~cm}-37 \%$ \\
\hline
\end{tabular}




\begin{tabular}{ll}
\hline & $\geq 10 \mathrm{~cm}-22.2 \%$ \\
& Median $-3($ range $0-35)$ \\
& $\leq 5-81.1 \%$ \\
Mitotic Figures $/ 50$ hpf (37) & $5-10-10.8 \%$ \\
& $\geq 10-8.1 \%$ \\
IHC: CD 117 status & Positive in $90.5 \%$ cases \\
IHC: DOG & 4 cases (all positive) \\
Outcomes & Major complications ( $\geq$ III) - $14.3 \%$ \\
Perioperative Events (30 days) & Mortality - $2.4 \%$ \\
Duration of Hospital stay & Median -8 days (range $1-38)$ \\
Follow up (36 cases) & Median -18 months (8- 42 months) \\
\hline
\end{tabular}

GIST- Gastrointestinal stromal tumor; $C E C T$ - Contrast enhanced computerized tomography; HPE- Histopathological examination; hpf- High power field; $I H C$ - Immunohistochemistry; DOG- Discovered on GIST.

Total of 42 cases of GIST was diagnosed pathologically over 36 months at TU teaching hospital (TUTH), Kathmandu. Patient clinical characteristics, tumor characteristics, and outcomes of a total of 42 cases of GIST were recorded in table 2 .

Diagnostic biopsies were done in 10 cases and definitive surgery performed in the remaining 32 cases. Out of 32,11 underwent conservative resections while 21 underwent extended resections. As part of definitive treatment, gastric GIST was managed with subtotal gastrectomy-2, partial gastrectomy-1, pylorus-preserving gastrectomy with gastrojejunostomy-1, wide local Excision-4, lap assisted distal gastrectomy-1, lap assisted wedge gastrectomy-3. Similarly, duodenal GIST was managed with Enucleation-2, Excision of D4 with side-side duodenojejunostomy-1, Whipple's pancreaticoduodenectomy (PD)-1 and Whipple's PD with left lateral sectionectomy-1. Regarding small bowel, 9 cases underwent resection of bowel with anastomosis while 1 case with partial excision. Large bowel (caecal) GIST managed with right hemicolectomy (1 open \& 1 lap assisted). Two omental GIST and one retroperitoneal GIST excised. In total 5 cases were managed with help of laparoscopy. Preoperative angioembolization was done in 3 cases (one gastric fundal GIST of size $30 \mathrm{~cm} \&$ two fundal GIST with bleeding). Similarly, neoadjuvant Imatinib was given in 2 cases one with D2 GIST with liver metastasis \& another gastric GIST along the lesser curvature. Total of 18 cases received adjuvant chemotherapy out of which 10 cases received as part of only palliative therapy. Major complications developed in $14.3 \%$ cases while one patient expired in 30 days perioperative period.

Risk categorization had been done using a modified NIH risk classification shown in figure 1.

Total of 36 cases was followed up with a median of 18 months revealed 4 expired and the remaining 32 had no issues. Out of 4 expired ones, 1 in-hospital mortality occurred on the $20^{\text {th }}$ postoperative day of gastric GIST, 2 cases of unresectable GIST (pancreatic tail with B/L adrenal and retroperitoneal) diagnosed with trucut- biopsy expired at 1.5 months and 6 months respectively follow up in which later had received imatinib also. The remaining one expired case was the case of preoperative diagnosis of the ileal stricture with post-radiation for carcinoma cervix with postoperative histopathology revealing GIST of ileum with recurrence noted at 5 months of follow up, and adjuvant not taken.

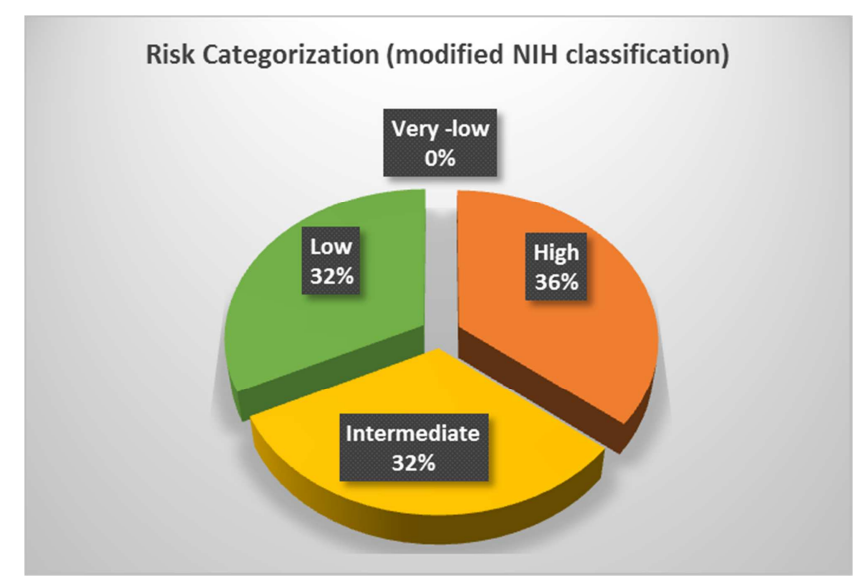

Figure 1. Risk categorization of 28 patients.

\section{Discussion}

Gastrointestinal stromal tumors (GISTs) are rare malignancies accounting for $1 \%$ to $2 \%$ of gastrointestinal neoplasms [11] but the most common mesenchymal tumors of the digestive tract [12]. Due to improvement in diagnostic imaging and frequent use of cross-sectional imaging, cases of GIST are encountered more frequently nowadays [12].

GISTs arise from interstitial Cajal cells which expresses CD117 (product of c-kit proto-oncogene), and harbors c-kit or gain-of-function mutation platelet-derived growth factor receptor alpha (PDGFRA) [13]. Grossly very wellcircumscribed fleshy, pink or tan-white mass is typical of GISTs. However, large tumors frequently present with necrosis, hemorrhage, and cystic degeneration. GISTs have three different histologic findings, including spindle (70\%), epithelioid (20\%), or mixed type (10\%).

Though male and female are equally affected and the peak incidence is in the $6^{\text {th }}$ decade, there is slight male preponderance in the study with a median age of 52 years. Stomach $(60-70 \%)$ is the most common location of GIST followed by small intestine, jejunum \& ileum (30\%), duodenum (5\%), rectum $(2-3 \%)$, colon ( $1-2 \%)$, esophagus $(<1 \%)$, omentum, mesentery, and peritoneum. Contrary to this, the stomach was found in $35.7 \%$ only while duodenum 
accounts $14.3 \%$ and no cases of rectal GIST were found. The higher number of duodenal GIST might be due to our hospital being tertiary level referral center. The most common symptoms of GISTs are gastrointestinal bleeding, including melena and hematemesis with subsequent anemia; weakness; and abdominal pain, distension, and discomfort due to mass effect [14].

Diagnosis is initially made by endoscopy, endoscopic ultrasound (EUS), or computed tomography (CT) of the abdomen [7]. CT was the most common diagnostic tool followed by exploratory laparotomy done for obstruction, peritonitis. CT is the gold standard for imaging that is used to characterize any abdominal mass in addition to assessing its extent and the presence/ absence of disease at a distance (GIST metastasize more frequently to the liver, omentum, and peritoneal cavity) [15]. Imaging reveals homogeneous densities in small tumors and larger tumors reveal irregular lobulated margins, mucosal ulceration, central and coagulative necrosis, hemorrhage cavitation, and heterogeneous enhancement [16]. A preoperative biopsy is not necessary for resectable GIST. But a biopsy is necessary when planning neoadjuvant imatinib therapy. Every attempt should be cared to avoid tumor spillage or metastasis. The EUS-guided biopsy is preferred to percutaneous biopsy [7]. However, a percutaneous image-guided biopsy may be allowed in metastatic diseases.

Pathological diagnosis of GIST is mainly based on the histological features but immunohistochemical (IHC) staining is needed to confirm the diagnosis [17]. CD117 is the most important IHC staining method for accurately diagnosing GIST in $94 \%-98 \%$ of patients. DOG1 (discovered on GIST) is a newly developed antibody (positive in $85 \%-95 \%$ of c-Kit-positive GISTs and in 30\%-36\% of c-Kit-negative GISTs) [18]. DOG-1 (Discovered on GIST-1) is a confirmatory IHC tool that solidifies the diagnosis when there is doubt [19]. Other IHC markers used are CD34, SMA, S-100, Desmin. CD117 positivity seen in $90.5 \%$ cases while 4 cases were diagnosed by DOG- 1 in this study.

As recommended by Asian GIST guidelines 2016, histopathological report for GIST should include details regarding (a) tumor location and size and mitotic index (per 50 high power fields); (b) resection margin status; (c) presence or absence of metastases or rupture during the operation; and (d) IHC staining results that include CD117 with or without CD34/DOG-1 [7].

As part of the optimal management of GIST, prediction of biological behavior is essential for the selection of patients for adjuvant therapy as well as guiding postoperative surveillance. But accurate prediction is not a straightforward algorithm. Various classifications have been developed like National Institutes of Health (NIH) consensus criteria [17], Armed Forces Institute of Pathology (AFIP) criteria [13], modified NIH classification [5]. Modified NIH classification [5] is more beneficial as it includes tumor size \& mitosis (NIH) and tumor location (AFIP) along with tumor rupture [20]. As per Asian guidelines published in 2016, modified
NIH criteria should be used to select the patients who will benefit from adjuvant targeted therapy [7]. On risk categorization of 28 patients, the majority fall in high risk $(36 \%)$ followed by intermediate (32\%) and low risk (32\%). No patients were categorized in a very low-risk group. As some patients underwent diagnostic biopsies only, complete risk categorization of all patients was not possible.

Smaller gastric tumors $(<2 \mathrm{~cm})$ without features of malignancy (such as irregular margin, ulceration, bleeding, cystic change, necrosis, or heterogeneous echogenicity in EUS) may be managed with active surveillance [7, 21]. However, a smaller size does not exclude the potential for malignancy and patients should be informed. For non-gastric GISTs, on the other hand, surgical resection is recommended, regardless of tumor size or morphology $[6,7]$. Complete en bloc resection with negative margin without a breach in the pseudo capsule should be the goal of surgical resection even in cases with tumor infiltration into the surrounding organs [7]. Omental and mesenteric GISTs should also be resected in bloc. Wedge resection of gastric GISTs and segmental resection of small bowel GISTs can be adequately provided to achieve negative margin. But simple enucleation or endoscopic treatment are not recommended even in smaller GISTs [7]. Reoperation is advisable whenever feasible if the macroscopic surgical margin positive (R2) [7]. However, cases of positive microscopic resection margin (R1), postoperative imatinib therapy can be considered based on malignant potential [7, 22]. Formal lymph node dissection is usually not necessary in GIST except in metastatic diseases [7]. Laparoscopic resection is safe and technically feasible for gastric GISTs $<5 \mathrm{~cm}$ as it is less invasive than open, with similar oncological yield $[6,23]$. However, laparoscopy is not recommended for GISTs $>5 \mathrm{~cm}$ or intestinal GISTs $[6,23]$.

Transcatheter arterial embolization (TAE) has proven to be a safe option for controlling gastrointestinal bleeding before attempting the best possible surgical treatment (preventing emergency surgery) [9]. We had performed TAE in 3 patients. Neoadjuvant therapy facilitates an R0 resection \& potential for organ preservation [7]. So neoadjuvant therapy has been advocated for rectum, the esophagus, GE junction, lesser curvature of the stomach, and in tumors with a high risk of rupture.

Targeted therapy has revolutionized the management of GIST [24]. Three years of adjuvant therapy proved superior to 1 year in modified NIH high-risk patients, with prolonged RFS and a slight benefit in OS [25]. Imatinib mesylate which is a selective tyrosine kinase inhibitor (TKI) of ABL, BCRABL, KIT, and PDGFR; initially approved for use in metastatic/ unresectable GIST in 2002 by FDA [7]. After 2008, its use had been approved for high-risk patients which later on got extended use approval after 2012. Imatinib has been shown to have a 50-67\% response rate, median PFS $>2$ yrs.; $15 \%$ with durable response $>10$ yrs. The usual dose is $400 \mathrm{mg} /$ day and the optimal duration is 3 years [7]. Other TKI includes Sunitinib useful in cases with imatinib-resistant GIST and Regorafenib useful as $3^{\text {rd }}$ line, surgical unresectable $\&$ refractory to other TKI. In targeted therapy, 
imatinib was given in 18 cases.

Follow up of the surgically treated patients is an important part of integrated management. GISTs with very low, low, and moderate risks are followed up by CT imaging every 6 months to 1 year, and high-risk and clinically malignant GISTs (with metastasis, breach to the pseudo capsule, peritoneal dissemination, or infiltration of adjacent organs) should be followed up by CT every 4 to 6 months [10]. Follow up were available in 36 patients with a median duration of 18 months.

Retrospective nature being one of the major limitations of the study. Some of the histopathological details are missing. Follow up was not possible in some of the patients. Long term follow-up is needed for evaluating the outcomes in terms of overall survival and recurrence-free survival.

\section{Conclusion}

GIST is not an uncommon disease. Clinical presentation is quite variable and symptoms are usually nonspecific \& depend on the size of the lesion and organ involvement. Majority of cases presented with pain abdomen and bleeding with location stomach, small bowel including duodenum. Surgery is the treatment of choice for patients with localized or potentially resectable GISTs. Ro excision is the goal with functional preservation whenever possible. Most of the patients were of the high-risk category. Targeted therapy (Imatinib) is the adjunct to multidisciplinary oncological management especially in high-risk patients and metastatic diseases. However, long-term follow-up (imaging, overall survival, and recurrence-free survival), adequate histopathological reporting is needed for future correlation.

\section{Conflicts of Interest}

Sah DN declares that there is no conflict of interest regarding the publication of this paper.

\section{References}

[1] H Joensuu, C Fletcher, S Dimitrijevic, et al. Management of malignant gastrointestinal stromal tumours. Lancet Oncol. 2002; 3 (11): 655-64.

[2] BP Rubin, MC Heinrich, CL Corless. Gastrointestinal stromal tumour. Lancet. 2007; 369 (9574): 1731-41.

[3] KM Kim, DW Kang, WS Moon, et al. Gastrointestinal stromal tumors in Koreans: it's incidence and the clinical, pathologic and immunohistochemical findings. J Korean Med Sci. 2005; 20 (6): 977-84.

[4] TM Parab, MJ DeRogatis, AM Boaz, et al. Gastrointestinal stromal tumors: a comprehensive review. J Gastrointest Oncol. 2019; 10 (1): 144-54.

[5] H Joensuu. Risk stratification of patients diagnosed with gastrointestinal stromal tumor. Hum Pathol. 2008; 39 (10): 1411-9.
[6] EESNWGJAooojotESfM Oncology. Gastrointestinal stromal tumours: ESMO Clinical Practice Guidelines for diagnosis, treatment and follow-up. 2014; 25: iii21.

[7] DH Koo, MH Ryu, KM Kim, et al. Asian Consensus Guidelines for the Diagnosis and Management of Gastrointestinal Stromal Tumor. Cancer Res Treat. 2016; 48 (4): 1155-66.

[8] RP Dematteo, JS Gold, L Saran, et al. Tumor mitotic rate, size, and location independently predict recurrence after resection of primary gastrointestinal stromal tumor (GIST). Cancer. 2008; 112 (3): 608-15.

[9] HJ Koo, JH Shin, S Shin, et al. Efficacy and Clinical Outcomes of Transcatheter Arterial Embolization for Gastrointestinal Bleeding from Gastrointestinal Stromal Tumor. J Vasc Interv Radiol. 2015; 26 (9): 1297-304 e1.

[10] K Akahoshi, M Oya, T Koga, Y Shiratsuchi. Current clinical management of gastrointestinal stromal tumor. World J Gastroenterol. 2018; 24 (26): 2806-17.

[11] MA Beltran, KSJIJoS Cruces. Primary tumors of jejunum and ileum as a cause of intestinal obstruction: a case control study. 2007; 5 (3): 183-91.

[12] A Aghdassi, A Christoph, F Dombrowski, et al. Gastrointestinal stromal tumors: clinical symptoms, location, metastasis formation, and associated malignancies in a single center retrospective study. 2018; 36 (5): 337-45.

[13] M Miettinen, J Lasota. Gastrointestinal stromal tumors: review on morphology, molecular pathology, prognosis, and differential diagnosis. Arch Pathol Lab Med. 2006; 130 (10): 1466-78.

[14] H Joensuu, P Hohenberger, CL Corless. Gastrointestinal stromal tumour. Lancet. 2013; 382 (9896): 973-83.

[15] JM Sanchez-Hidalgo, M Duran-Martinez, R Molero-Payan, et al. Gastrointestinal stromal tumors: A multidisciplinary challenge. World J Gastroenterol. 2018; 24 (18): 1925-41.

[16] J Gong, W Kang, J Zhu, J Xu. CT and MR imaging of gastrointestinal stromal tumor of stomach: a pictorial review. Quant Imaging Med Surg. 2012; 2 (4): 274-9.

[17] CD Fletcher, JJ Berman, C Corless, et al. Diagnosis of gastrointestinal stromal tumors: a consensus approach. 2002; 10 (2): 81-9.

[18] B Liegl, JL Hornick, CL Corless, CDJTAjosp Fletcher. Monoclonal antibody DOG1. 1 shows higher sensitivity than KIT in the diagnosis of gastrointestinal stromal tumors, including unusual subtypes. 2009; 33 (3): 437-46.

[19] AE Bannon, LR Klug, CL Corless, MCJEromd Heinrich. Using molecular diagnostic testing to personalize the treatment of patients with gastrointestinal stromal tumors. 2017; 17 (5): 445-57.

[20] P Rutkowski, E Bylina, A Wozniak, et al. Validation of the Joensuu risk criteria for primary resectable gastrointestinal stromal tumour-the impact of tumour rupture on patient outcomes. 2011; 37 (10): 890-6.

[21] JY Blay, S Bonvalot, P Casali, et al. Consensus meeting for the management of gastrointestinal stromal tumors. Report of the GIST Consensus Conference of 20-21 March 2004, under the auspices of ESMO. Ann Oncol. 2005; 16 (4): 566-78. 
[22] MD McCarter, CR Antonescu, KV Ballman, et al. Microscopically positive margins for primary gastrointestinal stromal tumors: analysis of risk factors and tumor recurrence. J Am Coll Surg. 2012; 215 (1): 53-9; discussion 9-60.

[23] M von Mehren, RL Randall, RS Benjamin, et al. Soft Tissue Sarcoma, Version 2.2016, NCCN Clinical Practice Guidelines in Oncology. J Natl Compr Canc Netw. 2016; 14 (6): 758-86.

[24] D Wang, Q Zhang, CD Blanke, et al. Phase II trial of neoadjuvant/adjuvant imatinib mesylate for advanced primary and metastatic/recurrent operable gastrointestinal stromal tumors: long-term follow-up results of Radiation Therapy Oncology Group 0132. Ann Surg Oncol. 2012; 19 (4): 107480 .

[25] H Joensuu, M Eriksson, K Sundby Hall, et al. Adjuvant Imatinib for High-Risk GI Stromal Tumor: Analysis of a Randomized Trial. J Clin Oncol. 2016; 34 (3): 244-50. 